\title{
Estudio in vitro de la resistencia a la tracción de postes de fibra de vidrio cementados con cuatro agentes cementantes
}

\section{In vitro study of push-out resistance of glass-fiber posts cemented with four luting agents}

Jara Vidal P*, Martínez Bello A**, Correa Beltrán G***, Catalán Sepúlveda A****

\section{RESUMEN}

Introducción: La causa más frecuente de fracaso de los pernos de fibra es el desprendimiento, debido a una falla de la adhesión en la interface dentina/agente cementante.

Objetivo: El objetivo de este estudio in vitro fue medir y comparar la resistencia a la tracción necesaria para producir la dislocación de postes de fibra de vidrio cementados con 4 agentes cementantes.

Materiales y métodos: Las coronas de 40 caninos sanos, fueron seccionadas transversalmente a nivel del techo cameral. Tras realizar el tratamiento endodóntico, los especímenes fueron mantenidos a $80 \%$ de humedad, a $37^{\circ} \mathrm{C}$ por 72 horas. Aleatoriamente se distribuyeron en 4 grupos $(n=10)$. Cada grupo recibió un poste de fibra de vidrio cementados con: Panavia F 2.0, Unicem, Fuji plus, Variolink. La cementación fue según indicaciones del fabricante y bajo presión digital, posteriormente se aplicó una carga estandarizada de 5 kilos por 5 minutos. Se efectuó una prueba de tracción utilizando una máquina de prueba universal con velocidad de $0,5 \mathrm{~mm} / \mathrm{min}$ hasta el descementado. A los datos se aplicó un diseño de Análisis de Varianza, ANOVA una Vía y el test de Tukey $(p<0,05)$.

Resultados: El coeficiente de determinación $\left(R^{2}\right)$ del modelo fue igual a un $88 \%$. La ANOVA mostró que al menos en uno de los cementos la media de la fuerza de tracción fue significativamente distinta a las demás $(p<0,0001)$. Al comparar las medias de la fuerzas de tracción se obtuvo diferencias estadísticamente significativas entre los 4 agentes cementantes $(p<0,05)$.

Este estudio in vitro demostró que el cemento de resina de curado-dual Panavia F 2.0 presentó la fuerza de tracción más alta. Por lo que en clínica se comportaría dentro de niveles muy aceptables.

Palabras clave: Cemento de resina, vidrio ionómero, perno de fibra de vidrio, fuerza de tracción.

\section{SUMMARY}

Introduction: The most frequent cause of the glass-fiber post failure was push-out due to a failure in the adhesion dentin/agent cement interface.

Purpose. The purpose of this in vitro study was to evaluate and compare the dislocation resistance of glassfiber post cemented by 4 different cement agents.

* DDS. Estudiante del Postgrado Especialización, Rehabilitación Oral Mención Prótesis. Departamento de Odontología Restauradora. Facultad de Odontología. Universidad Concepción. Concepción. Chile.

** DDS. Profesor Titular de Patología Oral, Departamento de Patología y Diagnóstico. Facultad de Odontología. Universidad de Concepción. Concepción. Chile.

*** MCS. Estadística. Departamento de Prevención y Salud Pública. Facultad de Odontología. Universidad de Concepción. Concepción. Chile.

**** DDS, MSC. Profesor Titular. Director Postgrado Rehabilitación Oral mención Prótesis. Departamento de Odontología Restauradora. Facultad de Odontología. Universidad Concepción. Concepción. Chile. 
Material and methods. Forty extracted canine were transversal sectioned at roof pulp level $(n=10)$. Each group received fiber posts were randomly cemented using cement Panavia F 2.0, Unicem, Fuji plus, Variolink. Each sample was loaded in tension in an Instrom universal testing machine. The maximum force required to dislodge each post was recorded. Data were subjected to 1-way ANOVA and Tukey tests. $(p<0.05)$.

Results. The determination coefficient $\left(R^{2}\right)$ of the model was $80 \%$. The one-way ANOVA disclosed significant differences between groups $(p<0.0001)$, and a multiple comparisons test revealed that statistically significant difference among the 4 cementing agents $(p<0.05)$.

Conclusions. This in vitro study demonstrated that dual-cured resin cement Panavia F 2.0 resulted in the highest dislodgement force thus would be within the clinically acceptable standard.

Key words: Dual-cured resin cement, ionomer cement, glass-fiber post, push-out, dislocation resistence.

Fecha de recepción: 17 de septiembre de 2009.

Aceptado para publicación: 2 de octubre de 2009.

Jara Vidal P, Martínez Bello A, Correa Beltrán G, Catalán Sepúlveda A. Estudio in vitro de la resistencia a la tracción de postes de fibra de vidrio cementados con cuatro agentes cementantes. Av. Odontoestomatol 2010; 26 (5): 255-262.

\section{INTRODUCCIÓN}

En la práctica clínica, los dientes endodónticamente tratados tienen a menudo una pérdida coronaria significativa y un compromiso de la estructura radicular del diente. Los factores responsables de este compromiso incluyen caries extensas, fracturas, traumas, iatrogenia, patología pulpar, como también tratamiento endodóntico $(1,2)$. Una manera rápida, sencilla y eficaz para solucionar los problemas de dientes endodónticamente tratados, es el empleo de coronas ancladas con pernos de fibra de vidrio $(3,4)$. Estos postes están constituidos de fibra de vidrio dispuesta de forma unidireccional en una matriz de resina. Resina de polímeros epóxicos con un alto grado de conversión del monómero y una estructura altamente reticulada, que utilizan adhesivos dentinarios basados en metacrilato y cementos de resinas (4-6). Una ventaja evidente de las fibras de vidrio es que distribuyen la tensión sobre una amplia área superficial, aumentando el umbral de la carga y reducción de fracturas radiculares $(1,2,5)$. Sin embargo, estudios recientes reportan que las restauraciones con postes de fibra pueden fracasar por dislocación de los postes (4). Estudios previos de la fuerza de unión y morfología han mostrado que la unión a los canales de la raíz pueden ser influencia- dos por procedimiento de endodoncia previo a la cementación del poste, por la variabilidad de dentina intrarradicular, por la compatibilidad de los cementos de resina, adhesivos dentinarios y el grosor de la película del agente cementante (1, 3, 4, 7-9).

Los agentes cementantes más comunes son fosfato de zinc, cementos de resina, vidrio ionómero, y cementos de vidrio ionómero modificados con resina. Los cementos de vidrio ionómero y vidrio ionómero modificados con resina se adhieren a la dentina vía mecanismos micro mecánicos y químicos (10), su contracción de fraguado se ve compensada por la expansión higroscópica post maduración $(4,11)$. La tendencia reciente ha estado hacia los cementos de resina, porque aumentan la retención y proporcionan por lo menos la consolidación a corto plazo de la raíz $(1,12)$. Los cementos basados en resina demuestran fuerzas iniciales mejores que los cementos de vidrio ionómero $(4,13,14)$, éstos se han empleado tradicionalmente para cementar los postes de la fibra (4). La unión entre el poste y la dentina intrarradicular, generalmente, se ve obstaculizada por las condiciones desfavorables que son inherentes dentro de los canales de la raíz $(4,15)$. La integridad de la unión es desafiada por la capacidad limitada de disipar las tensiones de contracción de la polimeriza- 
ción (16), en los espacios estrechos y largos que exhiben una geometría altamente desfavorable de la cavidad $(4,17)$. Seleccionar un cemento y el procedimiento apropiado para unir los postes a la dentina de la raíz es otro desafío. Se espera que el sellado sea fuerte debido a las mejoras recientes en la capacidad de sellar de los agentes cementante de resina adhesiva $(5,11)$. La causa más frecuente de fracaso de los pernos de fibra es el despegamiento, debido a un fracaso de la adhesión en la interfase dentina y el cemento de la resina $(1,4,18)$.

El objetivo de este estudio in vitro fue medir y comparar la fuerza de tracción necesaria para producir la dislocación de postes de fibra de vidrio cementados con 4 agentes cementantes. En el presente estudio se formula como hipótesis de trabajo que al menos uno de los cementos requiere una fuerza de tracción diferente para producir la dislocación del poste.

\section{MATERIALES Y MÉTODOS}

\section{Muestras}

Fueron seleccionados 40 caninos sanos. Las coronas clínicas fueron seccionadas transversalmente a nivel del techo de la cámara pulpar, con la ayuda de un disco de carburundum. Se efectuó el tratamiento de endodoncia en todos los dientes. Los conductos de las piezas dentarias fueron instrumentados manualmente hasta 1 milímetro sobre el foramen apical, con limas-K del 15-40 (lot 892140) y del 45-80 (lot 2313910) (Dentsply Maillefer, Ballaigues, Suiza) hasta un lima apical maestra \# 50 con la técnica estandarizada. Durante la instrumentación, los canales fueron irrigados con el hipoclorito del sodio al $2,5 \%$ y los conductos fueron secados con algodón absorbente.

Posteriormente los conductos fueron obturados con puntas maestras de gutapercha (Dentsply-Maillefer, Ballaigues, Suiza) calibre 55 y sellador permanente Tubli Seal (SybronEndo, Glendora, CA, Estados Unidos) usando la técnica de condensación lateral en frío con conos accesorios de gutapercha calibre 35 . El exceso extracoronal de gutapercha fue eliminado con un condensador caliente, luego las piezas fue- ron selladas con cemento provisional (Coltosol ${ }^{\circledR}$ Còltene/Whaledent, Altstätten, Suiza).

Los especímenes fueron mantenidos en un ambiente con un $80 \%$ de humedad y $37^{\circ} \mathrm{C}$ por 72 horas. Luego, el cemento provisional que selló los canales fue retirado usando una fresa redonda de carbide con una pieza de mano de baja velocidad (NSK Tokio, Japón). La preparación del conducto de la raíz para la inserción del poste de fibra de vidrio Ângelus (Ind. De Productos Odontológicos S/A Londrina PR, Brasil) fue iniciada con condensadores calentados para quitar parte del gutta-percha del inicio del conducto. La preparación fue terminada con un contraángulo de baja velocidad (NSK Tokio Japón), usando fresas largo secuenciales número 1, 2 y 3 (Dentsply Maillefer, Ballaigues, Suiza). Para determinar la extensión vertical de $10 \mathrm{~mm}$ en todas las muestras, se usó un tope de plástico, el ancho fue de 1,5 $\mathrm{mm}$ que quedo estandarizado por el diámetro de las fresas.

Una vez finalizada la preparación de los conductos, los dientes fueron distribuidos en forma aleatoria en 4 grupos de 10 muestras cada uno.

Los materiales cementantes testados son separados en los siguientes grupos:

I. Grupo GC Fuji Plus Reinforced Glass Ionomer Luting Cement GC Corporation (Tokio, Japón).

II. Grupo Panavia F 2.0 (Kuraray Medical INC, Okoyamo, Japón).

III. Grupo Unicem. 3 ESPE Dental Products (Seefeld, Alemania).

IV. Grupo Variolink II Professional set (Shaan, Liechtenstein).

Se procedió a la cementación con los distintos sistemas de cementado según las recomendaciones indicadas por el fabricante. La cementación fue bajo presión digital, eliminando el cemento sobrante en las áreas marginales con una sonda de caries curva. Posteriormente se aplicó una carga estandarizada de 5 kilos por 5 minutos. La polimerización del cemento en los casos en que era necesario se realizó por el método de foto polimerización (intensidad media de la luz $950 \mathrm{~mW} / \mathrm{cm}^{2}$ ), empleando LEDs de foto polimerización Smartlite ${ }^{\mathrm{TM}}$ PS, (Densply, De 
Trey GMBH, Alemania) con una duración de 60 segundos.

Después de 48 horas de almacenamiento de las muestras a $37^{\circ} \mathrm{C}$ con una humedad $85^{\circ}$, fueron sometidas a tracción con una velocidad de 0,5 mm/ min en la Máquina Universal de Ensayos para Tracción de $3.000 \mathrm{Kg}$ (Instron, modelo 4467 Londres, Inglaterra).

\section{Análisis estadístico}

Se utilizó el Programa SPSS versión 14.0 (Chicago, ILL, Estados (Inidos), se aplicó un diseño de Análisis de Varianza de una Vía. Cuyo factor fue el agente cementante con 4 niveles (I: Fuji plus; II: Panavia F2.0; III: Unicem; IV: Variolink). La variable respuesta es la fuerza de tracción en kilogramos. Las comparaciones múltiples fueron evaluadas con el test de Tukey. El nivel de significancia fue $p<0,05$.

\section{RESULTADOS}

En el diagnóstico del modelo, se revisaron los supuestos asociados y no se encontró evidencia para rechazarlos $(p=0,3283$, Shapiro-Willks; $p=0,7292$, Bartlett).

El coeficiente de determinación $\left(R^{2}\right)$ del modelo fue igual a un $88 \%$. La ANOVA que se presenta en la Tabla 1 muestra que al menos en uno de los cementos la media de la fuerza de tracción es significativamente distinta a las demás $(p<0,0001)$. Al comparar las medias de la fuerza de tracción se obtuvo diferencias estadísticamente significativas entre los 4 agentes cementantes ( $p<0,05$, Tukey). El agente cementante que necesitó una mayor fuerza de tracción fue Panavia F $2(43,08 \pm 3,47 \mathrm{Kg})$ y el que necesitó menor fuerza de tracción fue Variolink (17,06 $\pm 2,61 \mathrm{Kg})$.

La Tabla 2 presenta los Intervalos, con un 95\% de Confianza, para la fuerza de tracción media de los agentes cementantes y en el Gráfico 1 se visualiza el resultado de estas comparaciones.

\section{TABLA 2.- FUERZA DE TRACCIÓN MEDIA DE LOS AGENTES CEMENTANTES. PANAVIA F 2.0, UINICEM, FUJI PLUS, VARIOLINK}

\begin{tabular}{|c|c|}
\hline Agente cementante & Medias (Kg) \\
\hline Variolink . & $17,06 \pm 2,61$ \\
\hline Fuji plus.. & $23,69 \pm 2,74$ \\
\hline Unisem .......... & $32,66 \pm 3,12$ \\
\hline Panavia F 2.0 & $43,08 \pm 3,47$ \\
\hline
\end{tabular}

Intervalos de confianza del $95 \%$

\section{DISCUSIÓN}

El fracaso más frecuente de los postes de fibra cementados es el desprendimiento (4). Esto es, habitualmente, el resultado de una falla de adhe-

\section{TABLA 1.- ANÁLISIS DE VARIANZA DE UINA VÍA (ANOVA) PARA LOS AGENTES CEMENTANTES.} PANAVIA F 2.0, UNICEM, FUJI PLUS, VARIOLINK

\begin{tabular}{|l|r|r|r|r|r|}
\hline Fuentes de variación & g. $\mathbf{l .}$ & $\begin{array}{c}\text { Suma de } \\
\text { cuadrados }\end{array}$ & $\begin{array}{c}\text { Cuadrados } \\
\text { medios }\end{array}$ & test F & p \\
\hline Agente cementante & 3 & $3.256,01$ & $1.085,34$ & 74,15 & $<0,0001^{*}$ \\
Error & 30 & 439,09 & 14,64 & & \\
Total & 33 & $3.695,10$ & & & \\
\hline
\end{tabular}




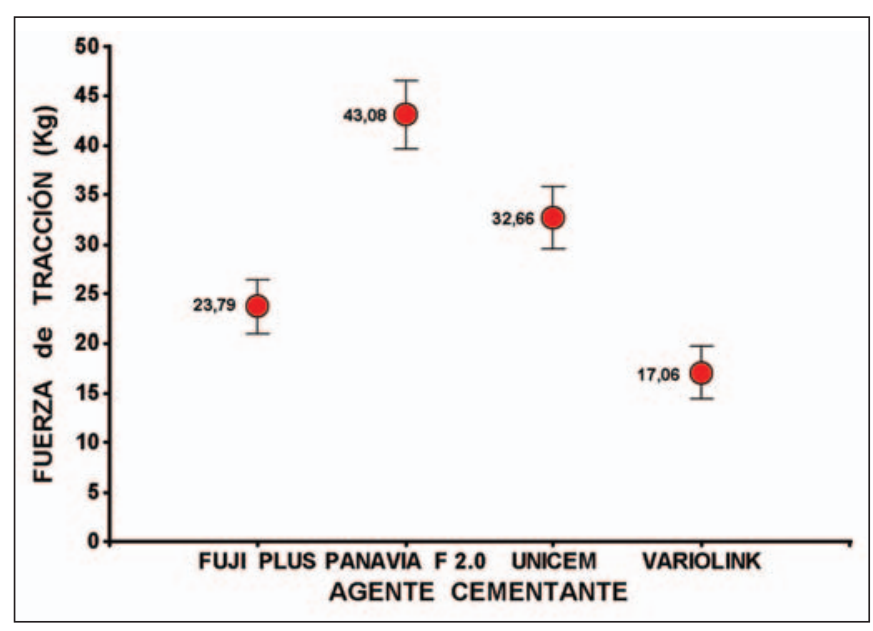

Fig. 1. Intervalos del 95\% de confianza de la Fuerza de tracción media de los cementos Panavia F 2.0, Unicem, Fuji plus y Variolink, expresadas en $\mathrm{Kg}$, medidas con la máquina Universal de Ensayos para Tracción. Las medias de los 4 agentes cementantes son estadísticamente diferentes ( $p<0,05$, Tukey).

sión en la interfase dentina y agente cementante $(1,4,18)$.

En el presente estudio in vitro se midieron y compararon las diferentes fuerzas de tracción necesarias para la dislocación de pernos de fibra de vidrio desde el conducto radicular cementados con Panavia F 2.0, Unicem, Fuji plus y Variolink. Los resultados de este estudio mostraron que hubo diferencias significativas en las fuerzas de tracción que se necesitaron para producir la dislocación del poste de fibra de vidrio desde el conducto radicular, entre los agentes cementantes Panavia F 2.0, Unicem, Fuji plus y Variolink.

Desde que se demostró en los estudios de push-out que el espesor del cemento de resina posee una influencia significativa en la fuerza de retención (19) es importante realizar el espacio necesario para el poste, usando las fresas indicadas, y distribuyendo el agente cementante con un léntulo, en los casos de Fuji plus y Variolink, procedimientos que se respetaron en la realización de esta investigación. El agente cementante Panavia F 2.0 no recomienda el uso del léntulo pues se aceleraría la polimerización; Unicem trae una punta de aplicación, lo que según Watzke y cols. (22) mejora y reduce la cantidad de imperfecciones en la interfase del cemento comparado con la técnica convencional.
Arcangelo y cols. (19) señalan que en varias investigaciones se revelan resultados controversibles concernientes a los valores de unión del poste al conducto radicular, debido a que la adhesión depende de varios factores que no son constantes y varía con el tipo de cemento utilizado y con la técnica de aplicación. Por otro lado, investigaciones recientes reportan que la retención del poste al conducto radicular se produce predominantemente por fricción $(6,19,20)$. Asmussen y cols. (9) demostraron que se requiere una fuerza de adhesión de $20 \mathrm{MPa}$ para compensar las fuerzas de contracción de la polimerización y lograr así una unión relativamente permanente entre el poste y la dentina radicular.

Los cementos resinosos adhesivos se unen a la dentina, a diferentes aleaciones metálicas como también a porcelana silanizada, y permiten conseguir una restauración intracoronal de gran retención, no generando grandes tensiones sobre el remanente radicular $(12,13,15,21)$. Varios estudios señalan que el uso de los cementos compuestos de resina han aportado una retención superior del poste $(12,13,15)$, hallazgos concordantes con los resultados de esta investigación, pues los cementos de resina con curado dual proporcionaron la máxima fuerza de adhesión, Panavia F 2.0 con 43,08 \pm 3,47 Kg y Unicem $32,66 \pm 3,12 \mathrm{Kg}$.

El agente cementante Panavia F 2.0 fue el que presentó un mejor comportamiento lo que concuerda con estudio O"Keefe y cols. (21) donde Panavia era muy superior a los cementos Bis-Core y C o B Metabond. Panavia está compuesto por bis-GMA y el monómero adhesivo 10-MDP (10-metacriloxidecil dihidrógeno fosfato), de manera que con una sola aplicación se efectúa el acondicionamiento dentinario (autograbante) y la adhesión a la dentina. Podría ser que esta dualidad de funciones que posee Panavia F 2.0 la que tendría un rol en lograr una fuerza de adhesión mayor que los otros cementos testados en este estudio.

El cemento Unicem al ser autoadhesivo y no necesitar grabado ácido ni la aplicación de un primer, facilita de manera importante su manipulación. En este estudio presentó el segundo mayor valor de retención de los cuatro agentes cementantes evaluados, 
resultados que están en conformidad con lo expresado por Watzke y cols. (22).

Según Cury y cols. (4) los cementos de vidrio ionómero, se han sugerido como alternativas para unir los postes de fibra a la dentina radicular con valores de retención en promedio de 8,9 MPa al test de pushout. Lo que se confirma en este estudio pues aunque el cemento Fuji plus testado no presentó el mejor resultado, y, en promedio soportó una fuerza de tracción de 23,69 $\pm 2,74 \mathrm{Kg}$ siendo más alto que la fuerza soportada por Variolink de 17,06 $\pm 2,61 \mathrm{Kg}$. A la luz de los resultados el cemento de vidrio ionómero reforzado con resina sigue siendo una alternativa para la cementación de pernos de fibra como ha sido demostrado en la literatura $(4,13,14)$.

Numerosos estudios de push-out indican que la adhesión de los postes de fibra de vidrio fracasa por la dislocación de este al conducto radicular (4, 23-25). Lo que se pudo comprobar según los resultados obtenidos en este estudio, en especial con el grupo Variolink, pues al traccionar con la máquina de ensayos Instron estos pernos salían con toda la capa de cemento adherida al poste, lo que indica que la adhesión del cemento hacia la dentina fue débil y no la del cemento al poste de fibra de vidrio.

Este estudio in vitro, muestra que los agentes cementantes de resina tuvieron mejores resultados que los de vidrio ionómero con la excepción de Variolink lo que se contrapone con lo demostrado por Radke y cols. (26), quienes reportaron que los cementos de zinc-fosfato y de vidrio ionómero son más retentivos que los cementos de resina. En cambio Goldman y cols. (27) obtuvieron valores retentivos más altos con cementos de resina que con cementos de fosfato o de vidrio-ionómero lo que está de acuerdo con los hallazgos del presente estudio.

Otras investigaciones muestran que los mejores resultados los obtuvo Variolink (3, 20, 28), lo que no está en concordancia con los hallazgos de este estudio. Esta discrepancia se podría explicar por la diferencia en el diseño del modelo de estudio, en la presente investigación se realizó traccionando el perno cementado en toda su extensión a la raíz del diente, en cambio en los otros estudios de push- out la raíz se secciona en tamaños de $1 \mathrm{~mm}$ y a estos trozos se les aplica fuerza para separar el perno de la raíz.

En este estudio in vitro, la diferencia más grande estuvo en dos cementos de resina con curado dual que fueron el Panavia F 2.0 con un sistema de autograbado presentando el mejor desempeño y Variolink con el peor. Esta situación se podría explicar por la estructura desfavorable de la dentina intrarradicular y, por la mayor complejidad en la manipulación del cemento Variolink.

Con las limitaciones de este estudio in vitro, donde se dejo sólo una condición diferente para cada grupo que era el cemento a usar, se puede decir que hubo diferencias estadísticamente significativas en la eficacia de los diferentes agentes cementantes del perno de fibra, por lo tanto se concluye que el cemento Panavia F 2.0 presenta una fuerza de unión mayor a los otros cementos testadas.

Basados en los resultados de este estudio se sugiere el uso de Panavia F 2.0 en el cementado de pernos de fibra de vidrio en el conducto radicular, ya que sería un procedimiento seguro y recomendable para uso clínico.

\section{CONCLUSIONES}

Dentro de las limitaciones de este estudio in vitro, se obtuvieron las siguientes conclusiones.

1. Panavia F 2.0 consiguió la fuerza de adhesión significativamente superiores a las de los otros cementos.

2. Panavia y Unicem obtuvieron fuerzas superiores a 32 kilos fuerza de tracción para desprenderse del conducto radicular.

3. Variolink aun siendo un cemento de resina de curado dual presentó el peor resultado.

4. Existieron diferencias estadísticamente significativas entre los cementos Panavia con los mejores resultados y con los cementos Unicem, Fuji plus y Variolink con los peores resultados. 


\section{AGRADECIMIENTOS}

Esta investigación fue soportada por el GRANT: Dirección de Investigación Universidad de Concepción DIUC -1.205.102.009-1.0.

\section{BIBLIOGRAFÍA}

1. Schwartz RS, Robbins JW. Post placement and restoration of endodontically treated teeth: a literature review. J Endod 2004;5:289-301.

2. De Almeida Conclaves LA, Vansan LP, Paulino $S M$, Sousa Neto MD. Fracture resistance of weakened roots restored with a transilluminating post and adhesive restorative materials: $\mathrm{J}$ Prosthet Dent 2006;96:339-44.

3. Goracci C, Sadek FT, Fabianelli FR, Ferrari M. Evaluation of the adhesion of fiber posts to intraradicular dentin. Operative Dentistry 2005;30(5):627-35.

4. Cury A, Goriacci C, de Lima Navarro M, Carvalho R, Sadek F, Tay F, Ferrari M. Effect of hygroscopic expansion on the push-out resisteance of glass ionomer-based cements used for the luting of glass fiber posts. 2006;32(6):537-40.

5. Kankan M, Usumez A, Oztururk AN, Belli S, Eskitascioglu G. Bond strength between root dentin and three glass-fiber post systems. J Prosthet Dent 2006;96:41-6.

6. Pirani Ch, Chersonui S, Foschi F, Piana G, Loushine RJ, Tay RR, Prati C. Does Hybridization of Intraradicular dentin really improve fiber post retention in endodontically treted teeth? J of Endodontic 2005;31:891-4.

7. Baldissara P, Zicari F, Valandro L, Scotti R. Effect root canal treatments on quartz fiber posts bonding to root dentin. J Endod 2006;32:985-88.

8. Akkayan B, Gülmez T. Resistance to fracture of endodontically treated teeth restored with different post systems. J Prosthet Dent 2002; 87:431-7.
9. Asmussen E, Peutzfeldt A, Heitmann T. Stiffness, elastic limit, and strength of newer types of endodontic posts. J Dent 1999;27:275-8.

10. Yiu CK, Tay FR, King NM, Pashley DH, Sidhu SK, Neo JC, Toledano M, Wong. Interaction of glassionomer cements with moist dentin. J Dent Res 2004;83:283-9.

11. Irie M, Suzuki K, Watts DC. Marginal and flexural integrity of three classes of luting cement, with early finishing and water storage. Dent Mater 2004;20:3-11.

12. Mezzomo E, Massa F, Libera SD. Fracture resistance of teeth restored with two different post-and-core designs cemented with two different cements: an in vitro study. Part I. Quintessence Int 2003;34:301-6.

13. Bachicha WS, Di Fiore PM, Miller DA, Lautenschlager EP, Pashley DH. Microleakage of endodontically treated teeth restored with posts. J Endod 1998;24:703-8.

14. Dauvillier BS, Feilzer A, de Gee AJ, Davidson CL. Visco-elastic parameters of dental restorative materials during setting. J Dent Res 2000;79: 818-23.

15. Morris MD, Lee KW, Agee KA, Bouillaguet S, Pashley DH. Effects of sodium hypoclorite and $\mathrm{RC}$-prep on bond strengths of resin cement to endodontic surfaces. J Endod 2001;27: 753-7.

16. Attar N, Tam LE, Mc Comb D. Mechanical and physical properties of contemporary dental luting agents. J Prosthet Dent 2003;89:127-34.

17. Schwartz RS, Fransman R. Adhesive dentistry and endodontics: materials, clinical strategies, and procedures for restoration of access cavities: a review. J Endod 2005;31:151-65.

18. Ferrari M, Vichi A, Mannocci F, Mason PN. Retrospective study of the clinical performance of fiber posts. Am J Dent 2000; 13 (Spec No): 9B-13B. 
19. Arcangelo CD, Amario MD, Vadini M, Zazzeroni S, Angelius FD, Caputi S. An evaluation of luting agent application technique effect on fibre post retention. J of Dentistry 2008;36:235-40.

20. Goracci C, Fabianelli A, Sadek FT, Papacchini F, Tay FR, Ferrari $M$. The contribution of friction to the dislocation resistance of bonded fiber posts. J Endod. 2005;31:608-12.

21. Okeefe KL, Miller BH, Powers JM. In vitro tensile bond strength of adhesive cements to new post materials. Int J Prosthodont 2000;13:47-51.

22. Watzke R, Blunck U, Frankenberger R, Naumann $M$. Interface homogeneity of adhesively luted glass fiber post. Dental Materials 2008;24:15127.

23. Grandini S, Sapio S, Goriacci C, Monticelli F, Ferrari $M$. A one step procedure for luting glass fibre posts: an SEM evaluation. Int Endod Journal 2004;37:672-8.

24. Vichi A, Grandini S, Ferrari M. Comparison between two clinical procedures for bonding fiber post into a root canal: A microscopic investigation. J of Endodontic 2002; 28:355-60.
25. Bittencourt Garrido AD, Sabbag Fonseca T, Alfredo E, Correa Silva-Sousa YT, Sousa-Neto MD. Influence of ultrasound, with and without water spray cooling, on removal of post cemented with resin or zinc phosphate cements. J of Endodontic 2004;30:173-6.

26. Radke RA, Barkhordar RA, Podesta RE. Retention of cast endodontic posts: comparison of cementing agents. J Prosthet Dent 1988;59:31820.

27. Goldman M, De Vitre R. Cement distribution and bond strength in cement posts. J Dent Res 1984; 63:1392-5.

28. Goracci C, Urbano A, Fabianelli A, Monticelli F, Raffaelini O, Cardoso PC, Tay F, Ferrari M. The adhesion between fiber posts and root canal walls: comparison between microtensile and push-out bond strength measurements. Eur J Oral Sci 2004;112:353-61.

\section{CORRESPONDENCIA}

Dr. Alfonso Catalán, DDS MSC

e-mail: acatalan@udec.cl 